\title{
Analysis of Ulysses Dust Measurements within the Asteroid Belt
}

I. Mann and M. Wilck

Max-Planck-Institut für Aeronomie, Postfach 20, D-37189

Katlenburg-Lindau, Germany

E. Grün

Max-Planck-Institut für Kernphysik, Postfach 1039 80, D-69029 Heidelberg, Germany

\begin{abstract}
Results of Ulysses impact measurements are compared to Zodiacal cloud models. We discuss the infiuence of radiation pressure forces on particles of different size and composition and show its relevance for the understanding of impact measurements, and give an upper estimate for the dust density in an IRAS dust band.
\end{abstract}

\section{Introduction}

The Ulysses dust measurements yield the possibility of comparing local dust flux rates with models of the spatial distribution of the interplanetary dust cloud. The latter are based on the analysis of Zodiacal light observations, describing particles in a size range from 1 to $100 \mu \mathrm{m}$. The impact experiment detects smaller particles, covering masses between $10^{-16}-10^{-7} \mathrm{~g}$, respectively $\mu \mathrm{m}$ to sub- $\mu \mathrm{m}$ size. Both dust components are mainly produced in a similar way from the collisional evolution of larger meteoritic particles which stem from cometary activity and collision of asteroids. Some of the forces, acting on dust particles, depend on their size and among them, especially the influence of radiation pressure is important for our case. We will discuss the effect of radiation pressure and moreover the geometric selection effects of the Ulysses dust detector in order to compare the data to results from brightness observations. The Ulysses data yield also the opportunity of a comparison to the IRAS dust band particles.

\section{Measurement}

The Ulysses dust measurements are discussed in detail by Grün et al. (1992) and Baguhl et al. (1992, see also this issue). They show a mainly constant particle flux within the ecliptic from about 2 AU outward to Jupiter. The flux close to $1 \mathrm{AU}$ is a little higher. There is no significant change of the mass distribution with solar distance (in this case we do not consider dust streams emitted from the Jupiter system neither do we consider interstellar dust particles.) The Zodiacal cloud models mentioned above usually describe the radial slope of number density as being proportional to about $1 / r$, as a result of the Poynting-Robertson 

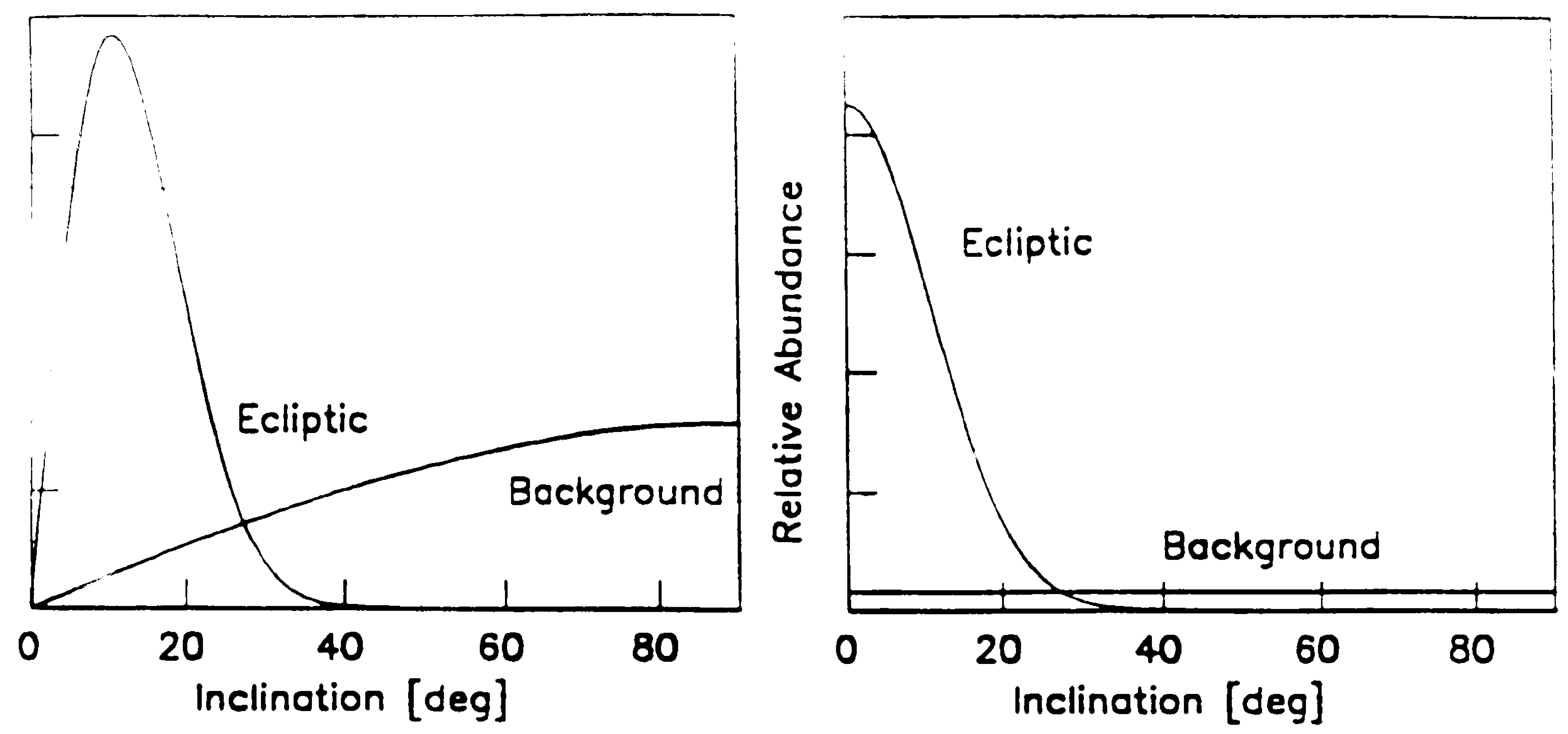

Figure 1. The distribution of inclinations according to a typical Zodiacal cloud model. The left figure shows absolute values. The right figure shows the same folded with the position probability in the ecliptic plane which is relevant for impact measurements.

deceleration of particles in circular orbits. However, different results exist about the outer solar system dust cloud. Also, the data include many particles in orbits inclined to the ecliptic plane, which is surprising, given the small probability of intercepting these particles in the ecliptic (see Figure 1).

\section{Influence of Radiation Pressure Forces}

Particles exposed to solar radiation in interplanetary space experience a radiation pressure force that depends on the energy flux of the radiation field as well as on the scattering process at the particle. It is directed away from the Sun and reduces the solar gravitational attraction. We have calculated the radiation pressure forces on dust particles in the mass range of $10^{-16}$ to $10^{-8} \mathrm{~g}$. This was based on Mie calculations applying the Maxwell-Garnett mixing rule to describe particles that consist of multiple components (see Wilck and Mann 1996). The results are presented in terms of values, $\beta=\mathrm{F}_{\text {grav }} / \mathrm{F}_{\mathrm{rad}}$, which denote the ratio of radiation pressure force $F_{\text {rad }}$ to gravitational force $F_{\text {grav }}$.

According to the discussion given by Fechtig (1989), we assume particles of cometary and asteroid origin and interstellar particles. In the case of the cometary dust we apply two types of models, one for very dark and porous particles, a second model is assumed to describe cometary dust that was processed in interplanetary space and got more compact. The calculations show that cometary dust particles, that are assumed to be very porous, suffer an especially high radiation pressure, which exceeds the gravitational solar attraction in a mass range from $10^{-15}$ to $10^{-12} \mathrm{~g}$. Interestingly, this strong influence of radiation pressure is only valid for this model assumption, whereas the other three discussed models show quite similar dependencies for the calculated $\beta$ values (see Figure 2). For large particles with masses $\mathrm{m}, 10^{-12} \mathrm{~g}<\mathrm{m}<10^{-8} \mathrm{~g}$, 


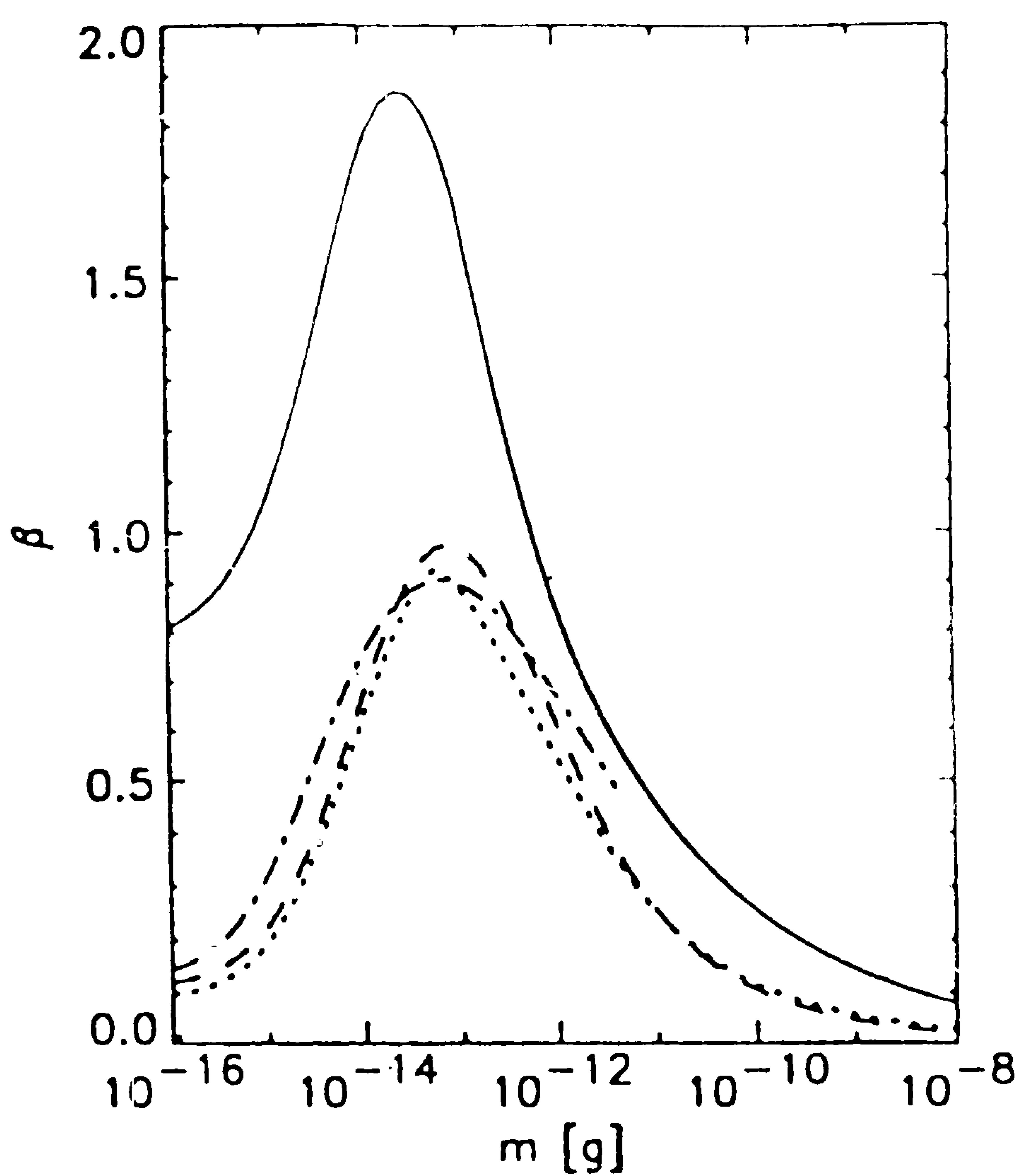

Figure 2. The calculated $\beta$-value versus size for four different models of dust particles, i.e. young cometary dust (solid line), evolved cometary dust (dotted line), asteroidal dust (dashed line) and interstellar dust (dashed - dotted line). Except for the young cometary dust, the calculated values are quite similar for different types of particles.

they follow the slope of the curve given for absorbing spheres. The $\beta$-values increase for smaller particles with a maximum between 0.85 and 0.95 for masses of $10^{-13} \mathrm{~g}$ and decrease again for smaller particles and are well below 0.3 at $10^{-15} \mathrm{~g}$ in all cases except for the young cometary dust.

\section{Geometric Selection Effects}

The calculations of the detection probability are presented in Figure 3. It shows the hypothetic dust flux (in arbitrary units) onto the detector given the parameters of the Ulysses orbit from 1 to $5.4 \mathrm{AU}$. We compare the fluxes for particles in low eccentricity low inclination orbits and particles in orbits with random inclination. It can be seen that the detection probability for the ecliptic component drops rapidly from $1 \mathrm{AU}$ outward and increases again around 4.5 AU But still, the isotropic component is detected predominantly, which explains the difference between Zodiacal cloud models and the present impact data. The comparison to the hypothetic flux estimated from an IRAS dust band (cf. Dermott et al. 1984) shows from the detection geometry, however, that these particles may be detected with a low efficiency. The number density of particles in an IRAS dust band can be estimated to amount to $\leq 8 \cdot 10^{-8} \mathrm{~m}^{-3}$ for particles of masses $\mathrm{m} \leq 10^{-11} \mathrm{~g}$ (Mann et al. 1996). Moreover, the dust measurements at larger distances are increasingly dominated by interstellar particles. We also checked the influence of the orbital eccentricity and the influence of the $\beta$-value. Even 

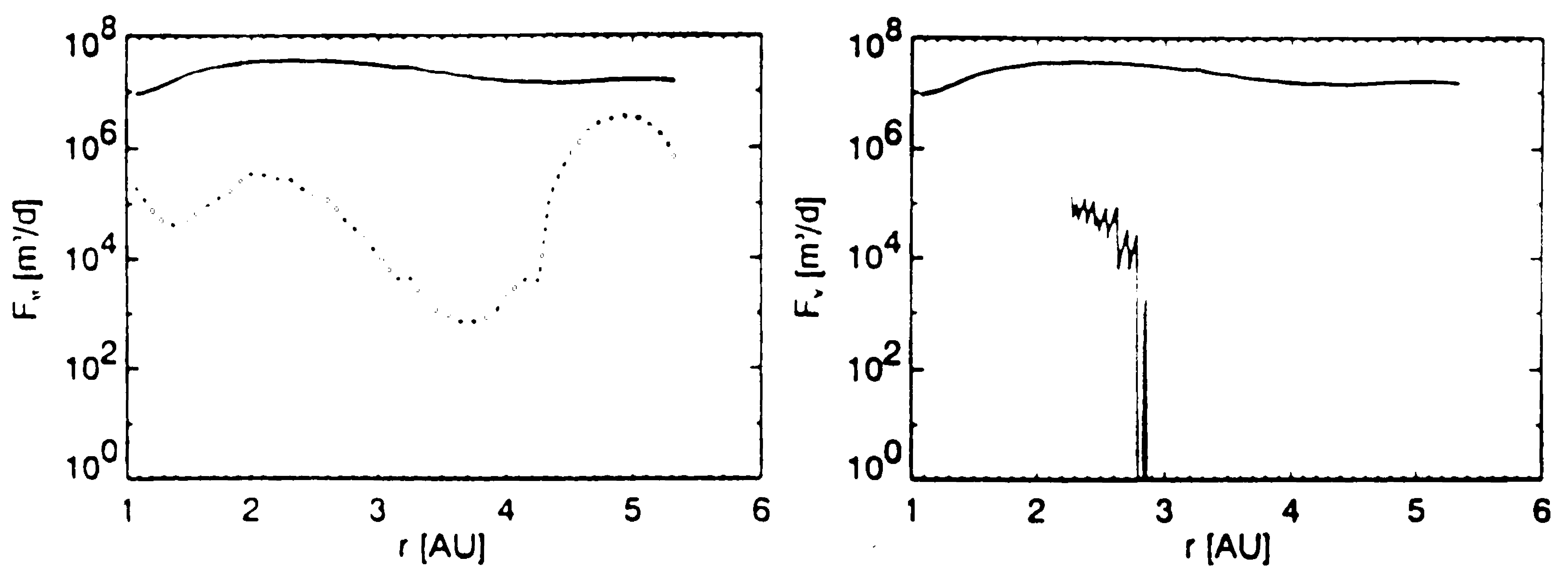

Figure 3. Hypothetic dust flux (in arbitrary units) onto the detector given the parameters of the Ulysses orbit from 1 to $5.4 \mathrm{AU}$. The left figure shows the hypothetic dust flux for particles in low eccentricity, low inclination orbits (dotted line) in comparison to particles in orbits with random inclination. All values are calculated with $\beta=0.1$. The right part shows the component of random inclination orbits in comparison with the hypothetic flux estimated from an IRAS dust band.

if the particles are in ecliptic (i.e. low inclination) orbits, they have a significant detection probability when the orbits have high eccentricities. This effect depends, as well, on the $\beta$-value.

\section{Conclusion}

As far as the analysis of impact data, especially the connection between impact velocities and particle orbits, is concerned the influence of radiation pressure forces is important for interplanetary dust particles. This influence varies with the size of the particles, however it could be shown that it is similar for different reasonable assumptions about the composition of the dust particles. The dust fluxes that were detected by Ulysses in its ecliptic orbit from the Earth to Jupiter are consistent with Zodiacal cloud models but biased by geometric selection effects.

\section{References}

Baguhl, M., Grün, E., Linkert, D., Linkert, G., \& Siddique, N. 1992, in Hypervelocity Impacts in Space, J. A. M. McDonnell, The Unit for Space Science, The University of Kent at Canterbury, 153

Dermott, S. F., Nicholson, P., Burns, J., \& Houck, J. 1984, Nature, 312, 505

Fechtig, H. 1989, Z. Naturforsch., 44a, 877

Grün, E., Fechtig, H., Giese, R. H., Kissel, J., Linkert, D., Maas, D., McDonnell, J. A. M., Morfill, G. E., Schwehm, G., \& Zook, H. A. 1992, A\&A, 92, 411

Mann, I., Grün, E., \& Wilck, M. 1996, Icarus, in press

Wilck, M., \& Mann, I. 1996, Planet. Space Sci., in press 